\title{
Emission Properties of Periodic Fast Radio Bursts from the Motion of Magnetars: Testing Dynamical Models
}

\author{
Dongzi $\mathrm{Li}^{1,2,3}$ (iD) and J. J. Zanazzi ${ }^{2}$ (i) \\ ${ }^{1}$ Cahill Center for Astronomy and Astrophysics, California Institute of Technology, 1216 E California Boulevard, Pasadena, CA 91125, USA; dongzili@ caltech.edu \\ ${ }^{2}$ Canadian Institute for Theoretical Astrophysics, University of Toronto, 60 St. George Street, Toronto, ON M5S 1A7, Canada \\ ${ }^{3}$ Department of Physics, University of Toronto, 60 St. George Street, Toronto, ON M5S 1A7, Canada \\ Received 2021 January 14; revised 2021 February 24; accepted 2021 March 1; published 2021 March 15
}

\begin{abstract}
Recent observations of the periodic fast radio burst source 180916.J0158+65 (FRB 180916) find small linear polarization position angle swings during and between bursts, with a burst activity window that becomes both narrower and earlier at higher frequencies. Although the observed chromatic activity window disfavors models of periodicity in FRB 180916 driven solely by the occultation of a neutron star by the optically thick wind from a stellar companion, the connection to theories where periodicity arises from the motion of a bursting magnetar remains unclear. In this Letter, we show how altitude-dependent radio emission from a magnetar, with bursts emitted from regions that are asymmetric with respect to the magnetic dipole axis, can lead to burst activity windows and polarization consistent with the recent observations. In particular, the fact that bursts arrive systematically earlier at higher frequencies disfavors theories where the FRB periodicity arises from forced precession of a magnetar by a companion or fallback disk, but is consistent with theories where periodicity originates from a slowly rotating or freely precessing magnetar. Several observational tests are proposed to verify/ differentiate between the remaining theories, and pin down which theory explains the periodicity in FRB 180916.
\end{abstract}

Unified Astronomy Thesaurus concepts: Compact radiation sources (289); Radio sources (1358); Neutron stars (1108); Radio bursts (1339); Magnetars (992)

\section{Introduction}

Fast radio bursts (FRBs) are short ( $\sim \mu$ s to $\sim$ ms duration) radio bursts with unknown origins. A decade after the first discovery (Lorimer et al. 2007), massive progress has been made in understanding the nature of FRBs. The discovery of repeating FRBs (e.g., Spitler et al. 2014, 2016; CHIME/FRB Collaboration et al. 2019; Fonseca et al. 2020) suggests at least a portion of the FRB population has a noncatastrophic origin. The first localization of an FRB source (Chatterjee et al. 2017) confirmed their cosmological origin. The detection of extremely bright radio bursts from a galactic magnetar (Bochenek et al. 2020; CHIME/FRB Collaboration et al. 2020b) places magnetars as a most promising progenitor for FRBs. Repeating FRBs offer great opportunities for follow-up observations, as well as studying the burst properties with time and frequency. FRB 180916.J0158+65 (hereafter FRB 180916) is the closest localized extragalactic FRB source $(z=0.0337$; Marcote et al. 2020), and also the most active repeating source detected within the frequency band $400-800 \mathrm{MHz}$ by the Canadian Hydrogen Intensity Mapping Experiment (CHIME; CHIME/ FRB Collaboration et al. 2019). Remarkably, a periodicity in the activity of FRB 180916 has recently been found. Bursts detected at $400-800 \mathrm{MHz}$ occur within a 5 day window that repeats every 16.3 days (CHIME/FRB Collaboration et al. 2020a). Multiple models have been proposed to explain the periodicity: the orbital motion of the FRB source (neutron star or magnetar) around a companion (Dai et al. 2016; Ioka \& Zhang 2020; Lyutikov et al. 2020), precession of the burst emitting object itself (e.g., Levin et al. 2020; Yang \& Zou 2020; Zanazzi \& Lai 2020), or ultralong rotational periods of the bursting object (Beniamini et al. 2020).

Multiwavelength follow-ups of this source provide constraints on the FRB progenitor and the local environment, and also introduce additional puzzles. Imaging from the Hubble Space Telescope shows that the location of FRB 180916 is offset by $\sim 250 \mathrm{pc}$ from the nearest knot of star formation in the host galaxy (Tendulkar et al. 2021), while young magnetars are expected to born in a star-forming region. For bursts detected in the $L$ band, the change of linear polarization position angle (PA) is constrained to be $\lesssim 10^{\circ}-20^{\circ}$ across the bursts of the same phase (Nimmo et al. 2020), and $\lesssim 50^{\circ}$ across the $L$-band active phases (Pastor-Marazuela et al. 2020). No PA swing is observed, as would generally be expected in the magnetospheric origin models (as opposed to the diverse polarization angle swing detected in FRB 180301; Luo et al. 2020). Most strikingly, the active phase is observed to be chromatic, with the activity window being both narrower and earlier at higher frequencies (Pastor-Marazuela et al. 2020; Pleunis et al. 2020). This disfavors models explaining the periodic activity with the eclipse of a companion wind, as these theories predict a narrower activity window at lower frequencies (Ioka \& Zhang 2020; Lyutikov et al. 2020).

Recently, a period of $\sim 160$ days was detected in the repeating FRB 121102 (Rajwade et al. 2020; Cruces et al. 2021). Similar to FRB 180916, FRB 121102 also has small PA swings during and between bursts (Michilli et al. 2018), but data on the frequency dependence of the activity window have yet to be gathered. Clearly, the polarization emission and frequency dependence expected from different models of periodic FRBs are becoming highly topical.

The goal of this work is to show how the recent constraints on the PA variation, as well as the dependence on the FRB activity window with frequency, fit into theories that argue the periodicity of FRB 180916 originates from the motion of a neutron star (NS) or magnetar. Section 2 justifies our phenomenological emission model for NSs emitting FRBs. Section 3 discusses the three dynamical theories that have been 

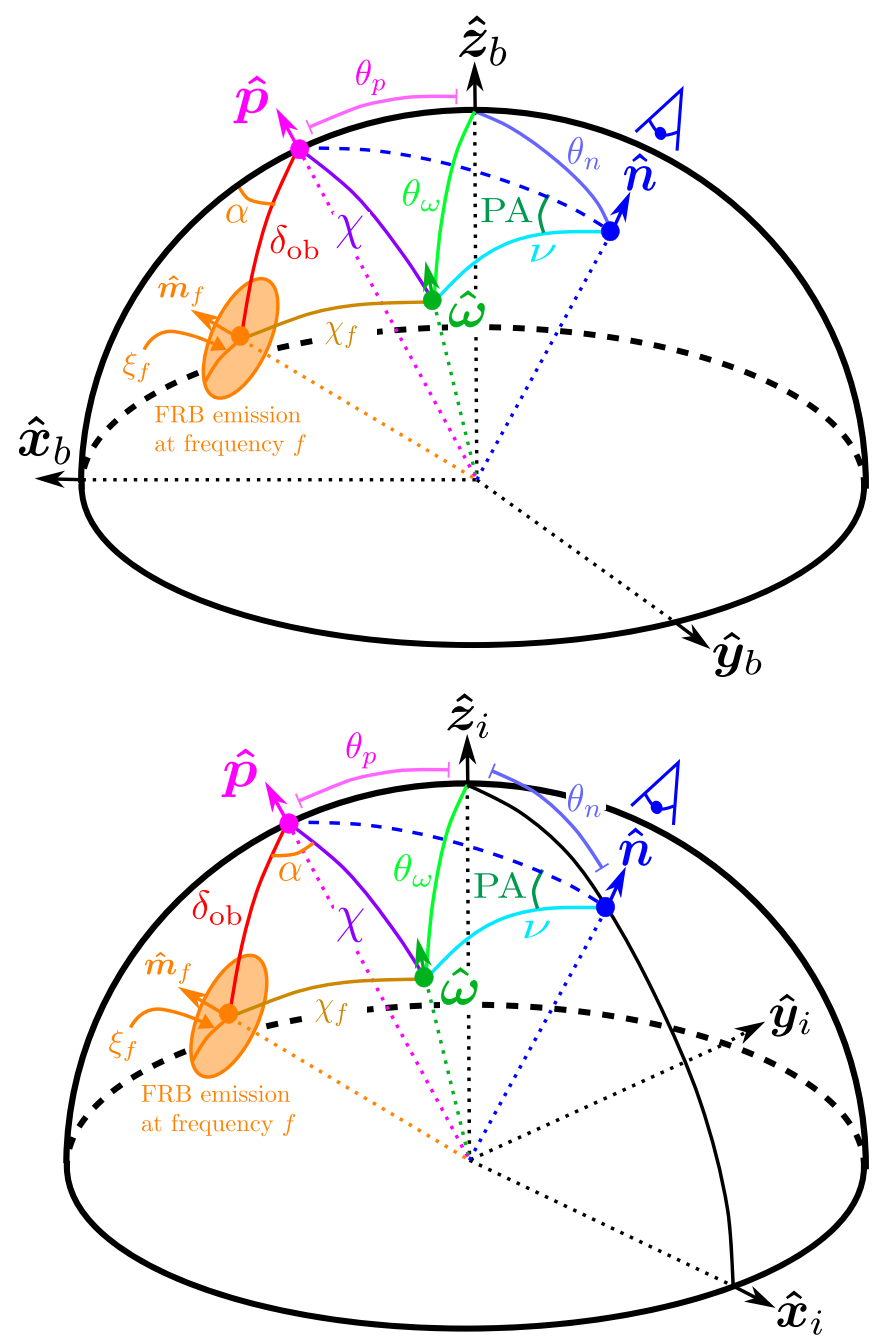

Figure 1. Geometry of our model for FRB emission in the upper half of an NS's magnetosphere. The FRB emission at frequency $f$ originates from a cone centered around $\hat{\boldsymbol{m}}_{f}$, with opening angle $\xi_{f}$. See Sections 2 and 3.3 for details. Top panel: model in the NS body frame $\left\{\hat{\boldsymbol{x}}_{b}, \hat{\boldsymbol{y}}_{b}, \hat{\boldsymbol{z}}_{b}\right\}$. Bottom panel: model in the NS inertial frame $\left\{\hat{\boldsymbol{x}}_{i}, \hat{\boldsymbol{y}}_{i}, \hat{z}_{i}\right\}$.

put forth to explain the periodicity of FRB 180916, which we test in this work: a magnetar with a long rotation period, an $\mathrm{NS}$ /magnetar undergoing free precession, and an NS/magnetar undergoing forced precession. Section 4 presents the main results of this work, and discusses which dynamical models are favored to explain the periodicity of FRB 180916. Section 5 discusses future observations that can potentially distinguish between the remaining theories. Section 6 summarizes our work.

\section{Emission Model for FRBs}

To make testable predictions for periodic FRB emission, we construct a phenomenological model of radio emission from a rotating (and possibly precessing) NS/magnetar, motivated by models for radio pulsar emission (e.g., Ruderman \& Sutherland 1975; Rankin 1993). Figure 1 displays the geometry of the model. An NS with rotation axis $\hat{\omega}$ and dipole moment unit vector $\hat{\boldsymbol{p}}$ is viewed by an observer, with $\hat{\boldsymbol{n}}$ being the unit vector pointing in the direction of the line of sight (LOS) of the observer. FRBs are emitted in a direction $\hat{\boldsymbol{m}}_{f}$ with frequency $f$, and an observer sees the FRB emission when $\hat{\boldsymbol{n}}$ lies within the cone centered on $\hat{\boldsymbol{m}}_{f}$ with opening angle $\xi_{f}$ (orange region of Figure 1). In the body frame $\left\{\hat{\boldsymbol{x}}_{b}, \hat{\boldsymbol{y}}_{b}, \hat{z}_{b}\right\}$ (top panel), the emission axis $\hat{\boldsymbol{m}}_{f}$ is offset from $\hat{\boldsymbol{p}}$ by a magnetic polar angle $\delta_{\mathrm{ob}}$ (angle between $\hat{\boldsymbol{m}}_{f}$ and $\hat{\boldsymbol{p}}$ ), and a magnetic longitude $\alpha$ (angle between planes spanned by vector pairs $\left\{\hat{\boldsymbol{x}}_{b}, \hat{\boldsymbol{p}}\right\}$ and $\left.\left\{\hat{\boldsymbol{m}}_{f}, \hat{\boldsymbol{p}}\right\}\right)$. The magnetic (angle between $\hat{\boldsymbol{p}}$ and $\hat{\boldsymbol{\omega}}$ ) and emission (angle between $\hat{\boldsymbol{m}}_{f}$ and $\hat{\boldsymbol{\omega}}$ ) angles are $\chi$ and $\chi_{f}$, respectively, while the angle between $\hat{\boldsymbol{n}}$ and $\hat{\boldsymbol{\omega}}$ is $\nu$. The angles between $\hat{\boldsymbol{p}}, \hat{\boldsymbol{\omega}}$, and $\hat{\boldsymbol{n}}$, and the symmetry axis $\hat{z}$, are $\theta_{p}, \theta_{\omega}$, and $\theta_{n}$, respectively. The position angle PA of the linear polarization (in the rotating vector model; Radhakrishnan \& Cooke 1969) is the angle between the planes spanned by the vector pairs $\{\hat{\boldsymbol{n}}, \hat{\boldsymbol{\omega}}\}$ and $\{\hat{\boldsymbol{n}}, \hat{\boldsymbol{p}}\}$.

The emission model assumed in this Letter is significantly more complex than the model in Zanazzi \& Lai (2020), which assumed $\hat{\boldsymbol{m}}_{f}=\hat{\boldsymbol{p}}$. In this section, we justify our model assumptions about the FRB emission region, assuming the coherent radio emission arises due to curvature radiation from charged particles traveling along the NS magnetic field lines. Magnetar curvature radiation has already been invoked by many models to explain the coherent radio emission characteristic of FRBs (e.g., Katz 2014; Cordes \& Wasserman 2016; Kumar et al. 2017; Lu \& Kumar 2018; Yang \& Zhang 2018; Lu et al. 2020; Yang et al. 2020). Section 2.1 discusses our expectations for how the emission direction $\hat{\boldsymbol{m}}_{f}$ changes with FRB frequency $f$, while Section 2.2 discusses the linear polarization of the emission.

\subsection{FRB Emission Direction}

In the study of pulsar emission, it is widely believed that different frequencies are produced at different altitudes within the pulsar magnetosphere (e.g., Manchester \& Taylor 1977). It has been shown that the spread of field lines at higher altitude could account for the widening of pulse profiles at lower frequencies (e.g., Cordes 1978; Phillips 1992). This kind of "radius-to-frequency mapping" has also been used to explain the downward-drifting pattern seen in some repeating FRBs (Wang et al. 2019; Lyutikov 2020). Without loss of generality, we demonstrate this same effect in the context of curvature radiation, and show how it will lead to the magnetic polar angle $\delta_{\text {ob }}$ and cone opening angle $\xi_{f}$ of the observed FRB emission to decrease with an increase in frequency $f$. Similar approaches could be applied to other FRB emission models expecting "radius-to-frequency mapping" (e.g., Wadiasingh \& Timokhin 2019).

For simplicity, we assume the magnetic field exterior to the NS is a dipole in vacuum, and ignore how plasma affects the magnetic field (e.g., Goldreich \& Julian 1969; Tchekhovskoy et al. 2016; Philippov \& Spitkovsky 2018). The trajectory of a single field line in polar coordinates is given by

$$
\frac{r}{R_{0}}=C \sin ^{2} \delta_{\mathrm{em}}
$$

where $\delta_{\mathrm{em}}$ is the magnetic polar angle of the field line, $r$ is the distance to the center of the NS, $R_{0}$ is the NS radius, while $1 \leqslant C<\infty$ is a constant that varies for different field lines. Emission at $\delta_{\text {em }}$ will be observed by a distant observer at a different magnetic polar angle $\delta_{\mathrm{ob}}$, which is related to $\delta_{\mathrm{em}}$ by

$$
\cos \delta_{\mathrm{ob}}=\frac{1+3 \cos 2 \delta_{\mathrm{em}}}{\sqrt{10+6 \cos 2 \delta_{\mathrm{em}}}} .
$$


The trajectory of a number of different magnetic field lines, as well as the relation between $\delta_{\mathrm{em}}$ and $\delta_{\mathrm{ob}}$, are displayed in the top panel of Figure 2.

The characteristic frequency $f$ of emission from curvature radiation is (e.g., Ruderman \& Sutherland 1975)

$$
f=\frac{3}{4 \pi} \gamma^{3} \frac{c}{\rho},
$$

where $\gamma$ is the Lorentz factor, while $\rho$ is the curvature radius. For a dipolar field (Equation (1)), $\rho$ can be shown to be (e.g., Yang \& Zhang 2018; Wang et al. 2019)

$$
\rho=\frac{r\left(1+3 \cos ^{2} \delta_{\mathrm{em}}\right)^{\frac{3}{2}}}{3 \sin \delta_{\mathrm{em}}\left(1+\cos ^{2} \delta_{\mathrm{em}}\right)} \equiv r F\left(\delta_{\mathrm{em}}\right) .
$$

Assuming for our simple model, the Lorentz factor decreases with $r$ as $\gamma(r)=\gamma_{0}\left(r / R_{0}\right)^{-2 / 3}$ (also assumed in, e.g., Lyutikov 2020 to explain the downward-drifting rate of FRBs), the emission frequency becomes

$$
f=K_{0} F\left(\delta_{\mathrm{em}}\right)\left(\frac{r}{R_{0}}\right)^{-3},
$$

where $K_{0}=3 c \gamma_{0}^{3} /\left(4 \pi R_{0}\right)$. Notice that a change from $r$ to $r^{\prime}$ can be compensated by a change of $K_{0}$ to $K_{0} r^{3} / r^{\prime 3}$ to emit in the same frequency.

Since we have specified a concrete radius-to-frequency mapping, our setup also allows us to calculate the frequency drift rate, due to different bust arrival times as the frequency emission region changes its location with respect to the NS surface. We follow the steps in Lyutikov (2020), assuming a stationary magnetosphere (i.e., the rotation is slow enough to be ignored during each burst). Assuming two subbursts observed at polar angle $\delta_{\mathrm{ob}}$ are initially triggered at the field lines $C_{1}, C_{2}$, at a distance $r_{0}$ from the NS surface, the time delay seen by the observer $\Delta t_{\mathrm{ob}}$ can be estimated with

$$
\Delta t_{\mathrm{ob}}=\frac{\Delta s}{v}-\frac{\Delta l}{c} .
$$

Here, $v \approx c\left(1-1 / 2 \gamma^{2}\right)$ is the speed of the moving particle, $\Delta l$ is the distance between the emitting point of the two field lines projected in the direction of LOS $\left(\delta_{\mathrm{em}}\right)$

$$
\Delta l=\left(r_{1}-r_{2}\right) \cos \left(\delta_{\mathrm{ob}}-\delta_{\mathrm{em}}\right),
$$

where $\delta_{\mathrm{em}}, C_{1}$, and $C_{2}$ together decide the emission heights $r_{1}$ and $r_{2}$ (Equation (1)). The quantity $\Delta s$ is the path difference along the two field lines. To calculate $\Delta s$, one first calculates the distance along the field lines from the triggering point to emitting point:

$$
s=\frac{r_{0}}{\sin ^{2} \delta_{0}} \int_{\delta_{0}}^{\delta_{\mathrm{em}}} \sqrt{1+3 \cos ^{2} \delta} \sin \delta d \delta,
$$

where the triggering magnetic polar angle angle $\delta_{0}$ is decided by $r_{0}$ and $C_{1} / C_{2}$ (Equation (1)). With this method, the time delay of subbursts, as well as the frequency drift rate, can be calculated. ${ }^{4}$

\footnotetext{
4 The rotation of the object can change the drift rate in both directions depending on the geometry (see, e.g., Figure 6 of Lyutikov 2020) and hence can increase the variation of the drift rate for a given source. However, the effect from rotation should not dominate the drift rate, otherwise there should also be upward-drifting bursts.
}
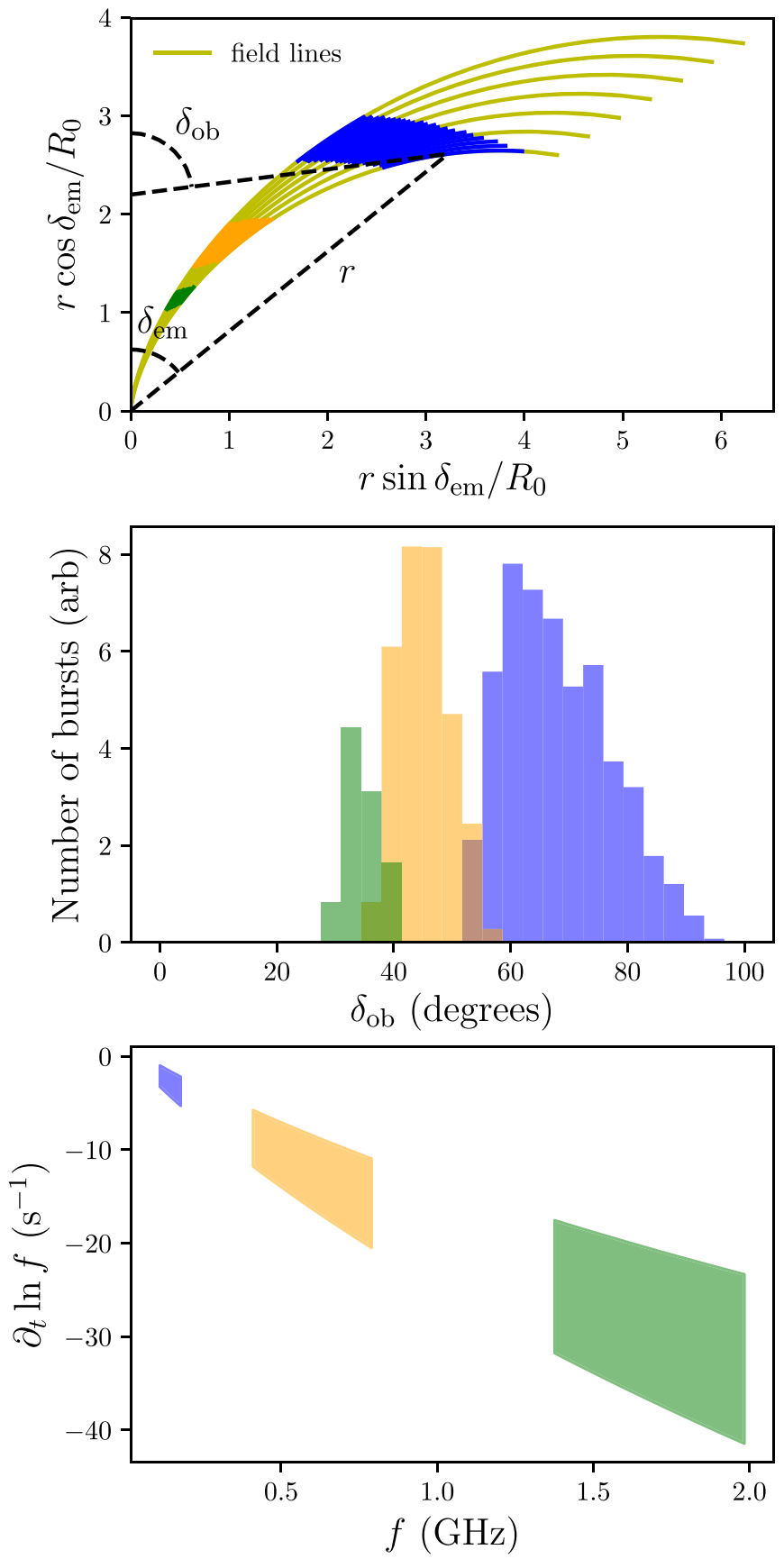

Figure 2. Altitude and angular dependence of FRB emission from curvature radiation (Equation (5)), for different frequency bands that are colored green $(1.36-2 \mathrm{GHz})$, orange $(400-800 \mathrm{MHz})$, and blue $(110-180 \mathrm{MHz})$, with $7 \leqslant C \leqslant 10$. Top panel: trajectories of magnetic field lines (Equation (1)). Field lines that cause emission in different frequency bands are colored. The magnetic polar angles $\delta_{\mathrm{em}}$ and $\delta_{\mathrm{ob}}$ are defined by the black dashed lines. Middle panel: histogram of observed magnetic polar angle $\delta_{\mathrm{ob}}$ of bursts from a centered dipole magnetic field. The histogram is computed assuming an equal number of bursts are emitted per unit length of the field line, with $C$ spaced linearly. The normalization for the number of bursts is arbitrary. The altitude of the burst emission, as well as the mean and variance of the burst emission $\delta_{\mathrm{ob}}$ values, increase with decreasing burst frequency $f$. Bottom panel: frequency drift rate of subbursts for a stationary magnetosphere, calculated using Equation (6) for the colored frequency bands. We assume that a group of emitting particles, disturbed at the surface of the magnetar, emit bursts at different heights after propagating some distance along the field lines. The downward drift in frequency is expected.

The top panel of Figure 2 shows the emission heights of different frequency bands, displayed in green $(1.36-2 \mathrm{GHz})$, orange $(400-800 \mathrm{MHz})$, and blue $(110-180 \mathrm{MHz})$. Here we 


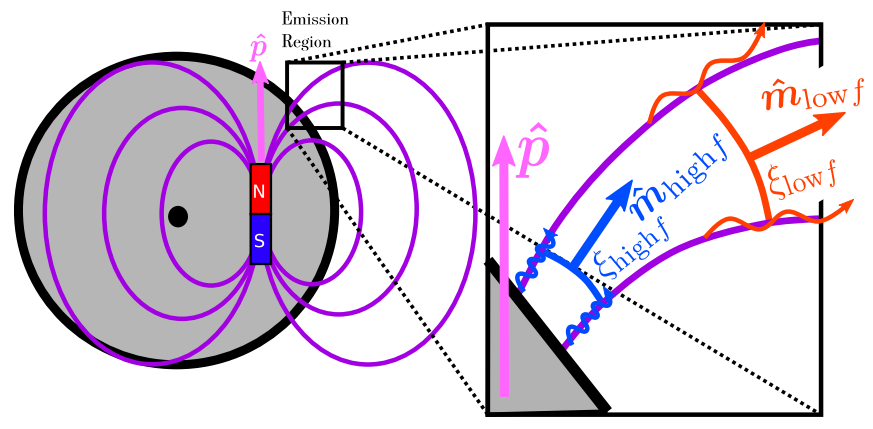

Figure 3. Cartoon representation of offset magnetic field geometry, which can give rise to FRB emission from curvature radiation consistent with our model (Figure 1). The strong exposed magnetic fields on one side of the NS lead to burst emission occurring in "cones" around the emission axis $\hat{\boldsymbol{m}}_{f}$, with both the magnetic polar angle $\delta_{\mathrm{ob}}$ (angle between $\hat{\boldsymbol{m}}_{f}$ and $\hat{\boldsymbol{p}}$ ) and opening angle $\xi_{f}$ increasing with decreasing frequency $f$.

take $R_{0}=10^{6} \mathrm{~cm}$ and $K_{0}=10 \mathrm{GHz}$ (corresponding to $\gamma_{0} \approx 100$ ). The emission height $r$ can be scaled with a change of $K_{0}$. Clearly for curvature radiation, high (low) frequency radiation originates at low (high) altitudes. The middle panel displays a histogram for a number of bursts with a given $\delta_{\mathrm{ob}}$ value, assuming an equal number of bursts are emitted per unit length of the field line, with the field line $C$ values spaced linearly between $7 \leqslant C \leqslant 10$. From this, we see both the mean and spread of $\delta_{\mathrm{ob}}$ values for a given frequency band increases with decreasing frequency. The bottom panel shows the frequency drift rate of subbursts for a stationary magnetosphere. We assume the emitting particles are disturbed at the magnetar surface (i.e., $r_{0}=R_{0}$ in Equation (8)). We calculate the drift rate for different observation angles $\delta_{\mathrm{ob}}$ shown in the middle panel, and display the range of the drift rate against frequency. The drift rate is always negative, which is consistent with the observed downward-drifting feature. (The rate can be adjusted by choosing a different $K_{0}$.) A scattering of $\gamma$ at the emission point, as well as a nonzero rotation during the subbursts, can increase the observed drift rate range.

Within this simple model, a dipole situated at the center of an NS will lead to FRB emission symmetric about the NS dipole moment $\hat{\boldsymbol{p}}$, rather than localized on a small "patch" at a location displaced from $\hat{\boldsymbol{p}}$. One simple modification to the magnetic field geometry that can lead to asymmetric emission about the $\hat{\boldsymbol{p}}$ axis is displayed in Figure 3: a dipole displaced from the NS center. The strong magnetic fields exposed on one side of the NS (which are buried on the other side in this cartoon) can lead to emission "cones" similar to those assumed in our model (Figure 1), with opening angles comparable to the width of the histogram widths displayed in the bottom panel of Figure 2. There is growing observational evidence many NSs may have similar magnetic field geometries as displayed in Figure 3. An offset dipole has been invoked to explain X-ray emission from the mode-switching pulsar PSR B0943+10 (Storch et al. 2014). Recently, X-ray observations from the NICER mission found the hot spots on the surface of isolated pulsars to be far from antipodal, implying a highly complex magnetic field far from the classic assumption of a centered dipole (Bilous et al. 2019; Riley et al. 2019; Kalapotharakos et al. 2021). For this work, rather than attempting to construct a complex magnetic field that can lead to FRB emission consistent with our model, we simply leave $\delta_{\mathrm{ob}}$ and $\xi_{f}$ as free parameters, with the general expectation both $\delta_{\mathrm{ob}}$ and $\xi_{f}$ should increase with decreasing $f$.

\subsection{FRB Polarization}

To model the polarization of FRB emission, we use the rotating vector model (Radhakrishnan \& Cooke 1969), where the position angle PA of the linear polarization is given by

$$
\tan \mathrm{PA}=\frac{-\sin \Psi \sin \chi}{\cos \chi \sin \nu-\cos \nu \sin \chi \cos \Psi},
$$

where $\Psi$ is the rotational phase ( $\Psi=0$ corresponds to when $\hat{\boldsymbol{n}}$ has its closest approach to $\hat{\boldsymbol{p}}$ ), while the other quantities are displayed in Figure 1. Although the magnetic field close to the NS may be complex, Lu et al. (2019) argued the propagation of a FRB across a plasma-filled NS magnetosphere causes the electric field of the burst to "freeze" in a direction perpendicular to the magnetic field when the plasma density is sufficiently low (and the magnetic field is approximately dipolar). Specifically, the electron-positron plasma number density $n_{p}$ must drop below (Equation (18) in Lu et al. 2019)

$$
\begin{aligned}
& n_{p} \lesssim \frac{1}{8 \pi^{2}} \sqrt{\frac{L}{r^{2} c}} \frac{1}{R_{\mathrm{B}}} \\
& =1.5 \times 10^{12}\left(\frac{10^{7} \mathrm{~cm}}{r}\right)\left(\frac{L}{10^{41} \mathrm{erg} \mathrm{s}^{-1}}\right)^{1 / 2}\left(\frac{10^{7} \mathrm{~cm}}{R_{\mathrm{B}}}\right) \mathrm{cm}^{-3}
\end{aligned}
$$

for PA to no longer change as the FRB propagates across the NS magnetosphere, where $L$ is the FRB equivalent isotropic luminosity, and $R_{\mathrm{B}}$ is the length scale over which the NS magnetic field locally varies. As long as condition (10) is met at the NS light-cylinder $R_{\mathrm{L}}=c / \omega$, where $\omega$ is the NS spin frequency, Equation (9) should be an adequate model for the linear polarization from FRBs. Other works have also used PA measurements to constrain the free precession of NSs (Weisberg et al. 2010). We neglect how additional propagation effects can cause the polarization to differ from the rotating vector model (e.g., Wang et al. 2010; Beskin \& Philippov 2012).

\section{Dynamics of Periodic FRB Models}

Models that ascribe the periodicity of FRB 180916 from the motion of a magnetar fall into three different categories. The simplest dynamical model postulated the 16.3 day periodicity was the rotation period of the magnetar (Beniamini et al. 2020), which implies this magnetar must have a rotation period much longer than those slow pulsars typically observed ( $\sim 0.1-10 \mathrm{~s}$; e.g., Kaspi \& Beloborodov 2017). Models that assumed more typical magnetar spin periods argued the periodicity of FRB 180916 came from either free (or "inertial") precession of the NS (Levin et al. 2020; Sob'yanin 2020; Zanazzi \& Lai 2020), or forced precession of the NS from a companion (Yang \& Zou 2020) or a fallback disk (Tong et al. 2020). In this section, we show how each scenario predicts different motions of a bursting magnetar with respect to a distant observer. Figure 4 summarizes how the motion of the NS spin axis $\hat{\omega}$, dipole moment axis $\hat{\boldsymbol{p}}$, emission direction axis $\hat{\boldsymbol{m}}_{f}$, and observer LOS axis $\hat{\boldsymbol{n}}$ differ between the three different dynamical theories. We defer a discussion of the physics that lead to these three different classes of magnetar motions to the references above.

The dynamics of these different motions are more conveniently analyzed in either a Cartesian coordinate system 

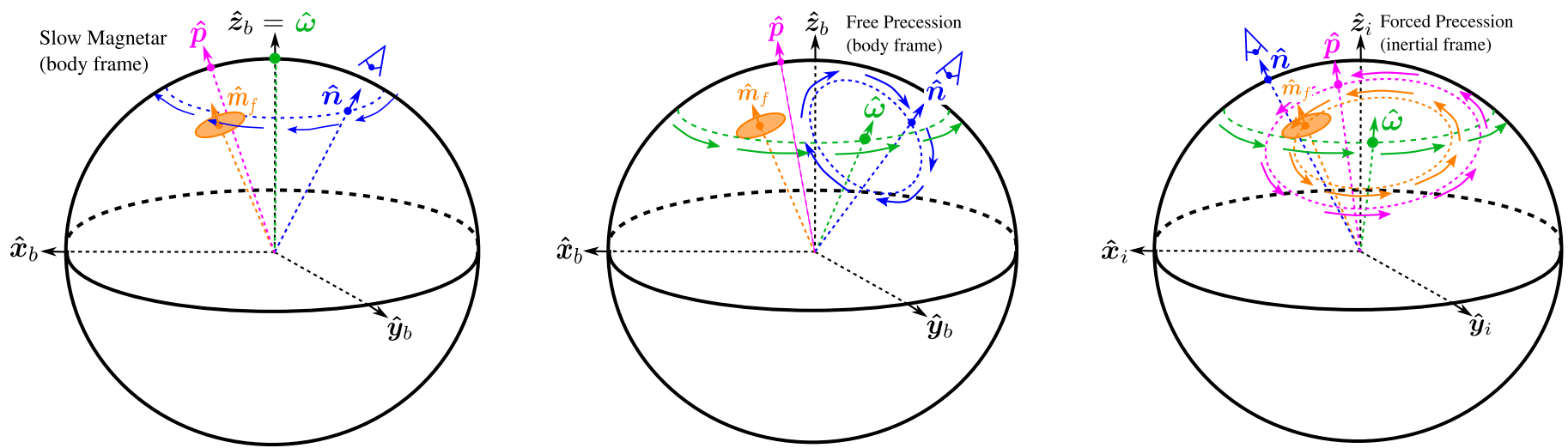

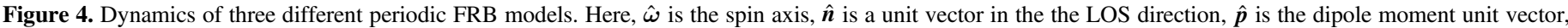

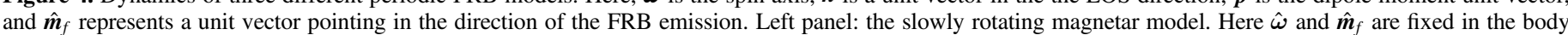

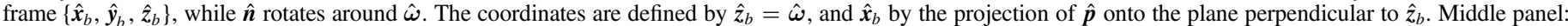

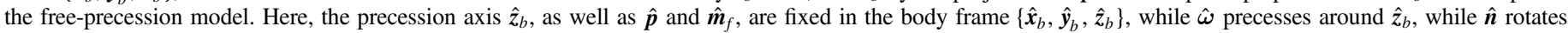

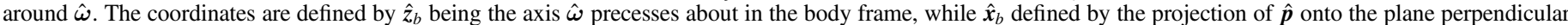

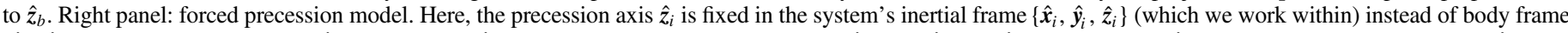

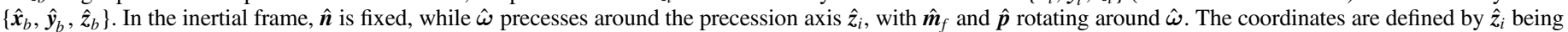
the axis $\hat{\boldsymbol{\omega}}$ precesses about, while $\hat{\boldsymbol{x}}_{i}$ defined by the projection of $\hat{\boldsymbol{n}}$ onto the plane perpendicular to $\hat{z}_{i}$.

anchored into and corotating with the NS $\left\{\hat{\boldsymbol{x}}_{b}, \hat{\boldsymbol{y}}_{b}, \hat{z}_{b}\right\}$ (body frame), or stationary with respect to a distant observer $\left\{\hat{\boldsymbol{x}}_{i}, \hat{\boldsymbol{y}}_{i}, \hat{\boldsymbol{z}}_{i}\right\}$ (inertial frame). The time evolution of a vector $\boldsymbol{v}(t)$ in the body $d v /\left.d t\right|_{b}$ or inertial $d v /\left.d t\right|_{i}$ frames are related via $d \boldsymbol{v} /\left.d t\right|_{b}+\boldsymbol{\omega} \times \boldsymbol{v}=d \boldsymbol{v} /\left.d t\right|_{i}$, where $\boldsymbol{\omega}=\omega \hat{\boldsymbol{\omega}}$ is the NS spin vector.

\subsection{Magnetar with Slow Rotation}

The left panel of Figure 4 displays our dynamical model for periodic FRBs due to slowly rotating NSs. We work in the body frame $\left\{\hat{\boldsymbol{x}}_{b}, \hat{\boldsymbol{y}}_{b}, \hat{\boldsymbol{z}}_{b}\right\}$, where $\hat{\boldsymbol{p}}$ and $\hat{\boldsymbol{m}}_{f}$ are static, with the (here static) rotation axis defining $\hat{z}_{b} \equiv \hat{\boldsymbol{\omega}}$, with $\hat{\boldsymbol{x}}_{b}$ lying in the direction of the projection of $\hat{\boldsymbol{p}}$ onto the plane perpendicular to $\hat{\boldsymbol{z}}_{b}$. Because $\hat{\boldsymbol{n}}$ is stationary in the inertial frame, it evolves as $d \hat{\boldsymbol{n}} /\left.d t\right|_{b}+\boldsymbol{\omega} \times \hat{\boldsymbol{n}}=0$ in the inertial frame, so

$$
\hat{\boldsymbol{n}}(t)=\sin \nu \cos \varphi_{n} \hat{\boldsymbol{x}}_{b}-\sin \nu \sin \varphi_{n} \hat{\boldsymbol{y}}_{b}+\cos \nu \hat{\boldsymbol{z}}_{b}
$$

with $\varphi_{n}(t)=\omega t+\varphi_{n}(0)$ as the spin phase. The spin phase is offset from the rotational phase $\Psi$ (see Equation (9)) by $\Psi=$ $\varphi_{n}+\pi / 2-\Delta_{n}$, where the offset angle $\Delta_{n}=\cos ^{-1}\left[\left(\cos \theta_{p}-\right.\right.$ $\left.\cos \theta_{\omega} \cos \chi_{)} /\left(\sin \theta_{\omega} \sin \theta_{p}\right)\right]$.

\subsection{Magnetar Undergoing Free Precession}

The middle panel of Figure 4 displays our dynamical model for a freely precessing NS. We work in the body frame, where the Cartesian coordinates $\left\{\hat{\boldsymbol{x}}_{b}, \hat{\boldsymbol{y}}_{b}, \hat{\boldsymbol{z}}_{b}\right\}$ define the (effective) principal axis of the biaxial NS, with $\hat{\boldsymbol{\omega}}$ precessing around $\hat{z}_{b}$ according to

$$
\hat{\boldsymbol{\omega}}(t)=\sin \theta_{\omega} \cos \varphi_{\omega} \hat{\boldsymbol{x}}_{b}+\sin \theta_{\omega} \sin \varphi_{\omega} \hat{\boldsymbol{y}}_{b}+\cos \theta_{\omega} \hat{\boldsymbol{z}}_{b},
$$

where $\varphi_{\omega}(t)=\Omega_{\text {prec }} t+\varphi_{\omega}(0)$ is the precession phase, with $\Omega_{\text {prec }}$ the precession frequency (see Zanazzi \& Lai 2015, 2020 for details). The vector $\hat{\boldsymbol{x}}_{b}$ lies in the direction of the projection of $\hat{\boldsymbol{p}}$ onto the plane perpendicular to $\hat{\boldsymbol{z}}_{b}$.

When the precession frequency is much smaller than the rotational frequency $\left(\Omega_{\text {prec }} \ll \omega\right)$, $\hat{\omega}$ remains approximately constant as $\hat{\boldsymbol{n}}$ rotates around $\hat{\boldsymbol{\omega}}$ over the rotational period of the NS (in the body frame). Hence $\hat{\boldsymbol{n}}(t)$ is described by an equation similar to (11), except in a frame where $\hat{z}_{b} \neq \hat{\omega}$ :

$$
\begin{aligned}
\hat{\boldsymbol{n}}(t)= & \frac{\sin \nu \cos \varphi_{n}}{\sin \theta_{\omega}}\left(\hat{\boldsymbol{z}}_{b} \times \hat{\boldsymbol{\omega}}\right) \times \hat{\boldsymbol{\omega}} \\
& -\frac{\sin \nu \sin \varphi_{n}}{\sin \theta_{\omega}}\left(\hat{\boldsymbol{z}}_{b} \times \hat{\boldsymbol{\omega}}\right)+\cos \nu \hat{\boldsymbol{\omega}},
\end{aligned}
$$

where $\varphi_{n}(t)=\omega t+\varphi_{n}(0)$ is the spin phase.

\subsection{Magnetar Undergoing Forced Precession}

The right panel of Figure 4 displays our model for forced precession, either by a companion (Yang \& Zou 2020) or a fallback disk (Tong et al. 2020). Figure 1, bottom panel, shows the system setup in the inertial frame of the observer $\left\{\hat{\boldsymbol{x}}_{i}, \hat{\boldsymbol{y}}_{i}, \hat{\boldsymbol{z}}_{i}\right\}$, with the orbital angular momentum axis of the companion or disk defining $\hat{z}_{i}$, with $\hat{\boldsymbol{x}}_{i}$ lying in the direction of the projection of $\hat{\boldsymbol{n}}$ onto the plane perpendicular to $\hat{\boldsymbol{z}}_{i}$. The magnetic longitude $\alpha$ is defined as the angle between the planes spanned by the vector pairs $\{\hat{\boldsymbol{p}}, \hat{\boldsymbol{\omega}}\}$ and $\left\{\hat{\boldsymbol{p}}, \hat{\boldsymbol{m}}_{f}\right\}$. All the quantities are illustrated in the bottom panel of Figure 1.

Assuming the spin angular momentum of the NS $\boldsymbol{L} \simeq I \boldsymbol{\omega}$, with $I$ the NS moment of inertia, the NS spin evolves in the forced precession theories according to an equation of the form $d \hat{\boldsymbol{\omega}} /\left.d t\right|_{i}-\Omega_{\mathrm{prec}} \hat{z}_{i} \times \hat{\boldsymbol{\omega}}=0$, and hence has a solution

$$
\hat{\boldsymbol{\omega}}(t)=\sin \theta_{\omega} \cos \varphi_{\omega} \hat{\boldsymbol{x}}_{i}+\sin \theta_{\omega} \sin \varphi_{\omega} \hat{\boldsymbol{y}}_{i}+\cos \theta_{\omega} \hat{z}_{i},
$$

where $\varphi_{\omega}(t)=\Omega_{\text {prec }} t+\varphi_{\omega}(0)$ is the precession phase. Because $\hat{\boldsymbol{p}}$ and $\hat{\boldsymbol{m}}_{f}$ are anchored in the rotating NS, they rotate in the inertial frame according to $d \hat{\boldsymbol{p}} /\left.d t\right|_{i}-\boldsymbol{\omega} \times \hat{\boldsymbol{p}}=0$ and $d \hat{\boldsymbol{m}}_{f} /\left.d t\right|_{i}-$ $\boldsymbol{\omega} \times \hat{\boldsymbol{m}}_{f}=0$. Since $\omega \gg \Omega_{\text {prec }}, \hat{\boldsymbol{\omega}}$ stays approximately constant as $\hat{\boldsymbol{p}}$ and $\hat{\boldsymbol{m}}_{f}$ rapidly rotate around $\hat{\boldsymbol{\omega}}$, hence the motion of $\hat{\boldsymbol{p}}(t)$ and $\hat{\boldsymbol{m}}_{f}(t)$ are approximately described by

$$
\begin{aligned}
\hat{\boldsymbol{p}}(t)= & \frac{\sin \chi \cos \varphi_{n}}{\sin \theta_{\omega}}\left(\hat{\boldsymbol{z}}_{i} \times \hat{\boldsymbol{\omega}}\right) \times \hat{\boldsymbol{\omega}} \\
& +\frac{\sin \chi \sin \varphi_{n}}{\sin \theta_{\omega}}\left(\hat{\boldsymbol{z}}_{i} \times \hat{\boldsymbol{\omega}}\right)+\cos \chi \hat{\boldsymbol{\omega}},
\end{aligned}
$$




$$
\begin{aligned}
\hat{\boldsymbol{m}}_{f}(t)= & \frac{\sin \chi_{f} \cos \varphi_{n}}{\sin \theta_{\omega}}\left(\hat{z}_{i} \times \hat{\boldsymbol{\omega}}\right) \times \hat{\boldsymbol{\omega}} \\
& +\frac{\sin \chi_{f} \sin \varphi_{n}}{\sin \theta_{\omega}}\left(\hat{\boldsymbol{z}}_{i} \times \hat{\boldsymbol{\omega}}\right)+\cos \chi_{f} \hat{\boldsymbol{\omega}},
\end{aligned}
$$

where $\varphi_{n}(t)=\omega t+\varphi_{n}(0)$ is the spin phase.

\section{Application of Periodic Emission Model to FRB 180916}

Now that we have developed a phenomenological emission model for periodic FRBs due to the motion of NSs, we apply these results to the recent multiwavelength and polarization measurements of FRB 180916. For a dynamical model to remain a plausible explanation for the periodicity of FRB 180916, it must explain the following three features of the FRB 180916 emission (Pastor-Marazuela et al. 2020; Pleunis et al. 2020):

1. The linear polarization angle PA varies by less than $\Delta \mathrm{PA} \lesssim 10^{\circ}-20^{\circ}$ for single bursts, and $\triangle \mathrm{PA} \lesssim 40^{\circ}$ between bursts.

2. The bust activity window (region in phase busts seen after folding over 16.3 day periodicity) widens with a decrease in frequency.

3. The burst activity windows in different frequency bands have phase centers that are offset from one another, or bursts at one frequency are systematically delayed with respect to bursts at another frequency. We will call this effect activity window phase drift with frequency.

The first constraint (with $\triangle \mathrm{PA}$ ) was at odds with the original predictions of the Zanazzi \& Lai (2020) free-precession model. This is because the emission region was assumed to emit in the direction of the NS dipole moment $\left(\hat{\boldsymbol{m}}_{f}=\hat{\boldsymbol{p}}\right.$; see Figure 1). Because FRBs occur when $\hat{\boldsymbol{n}} \approx \hat{\boldsymbol{m}}_{f}=\hat{\boldsymbol{p}}$, the rotational phase $\Psi \ll 1$ during an FRB, implying Equation (9) reduces to

$$
\tan \mathrm{PA} \simeq \frac{\sin \chi}{\sin (\chi-\nu)} \Psi
$$

Since $\hat{\boldsymbol{n}} \approx \hat{\boldsymbol{p}}$ implies $\chi \approx \nu$ (see Figure 1 ), the denominator of Equation (17) becomes small, and hence the model of Zanazzi \& Lai (2020) predicted large variations in PA during individual FRB bursts, as well as modulation of the PA variation between bursts as the NS precessed. However, because $\hat{\boldsymbol{m}}_{f}$ and $\hat{\boldsymbol{p}}$ can have significant differences in orientation (see Section 2.1), it is possible for PA variations to be sufficiently small to be consistent with the polarization measurements from FRB 180916.

In this section, we calculate the variations in PA, as well as how the burst activity window depends on frequency $f$, with different dynamical models (Figure 4). We create maps of the range in PA variation, and estimate the activity window of different models, by first fixing parameters that are constant for a given dynamical model (see Figure 1), and in particular specifying $\hat{\boldsymbol{m}}_{f}$ and $\xi_{f}$ for different frequency bands. Notice here that we assume the shift of emission region in the observed polar angle $\delta_{\mathrm{ob}}$ while the center of the emission region has the same magnetic longitude $\alpha$. A burst is considered observed at a specific $f$ band if $\hat{\boldsymbol{n}}$ and $\hat{\boldsymbol{m}}_{f}$ lie within an angle $\xi_{f}$ of each other, with no emission if this condition is not met. The phase $=\left(\varphi_{n}+\Phi\right) /(2 \pi)$ for the slow magnetar model, phase $=\left(\varphi_{\omega}+\Phi\right) /(2 \pi)$ for the free/forced precession model (with $\Phi$ a constant picked so the $400-800 \mathrm{MHz} f$ band is centered at phase $=0.5)$, and spin phase $=\varphi_{n} /(2 \pi)$ are then cycled through their possible parameter values $\left(\varphi_{\omega}, \varphi_{n} \in\right.$ $[0,2 \pi]$; middle panels of Figure 5) to calculate the range of PA values with (spin or precession) phase (top panels of Figure 5). The number of bursts over all spin phases are then binned by precessional phase to construct burst activity window profiles (bottom panels of Figure 5).

The top panels of Figure 5 display the PA variation with either spin (left panels) or precession (middle and right panels) phase. From this, we see all three models are able to have PA variations consistent with observations, as long as the emission region direction $\hat{\boldsymbol{m}}_{f}$ has a significant offset from the dipole axis $\hat{\boldsymbol{p}}\left(\delta_{\text {obs }}\right.$ sufficiently large $)$. The spin phase has similar variations as the PA with spin or precession phase, with differences due to the slight differences in geometry between the two angles (see Equation (9)).

The bottom panels of Figure 5 display the activity window over different $f$ bands. The magnetar with a slow rotation period, as well as the magnetar undergoing free precession, can accommodate an activity window that widens at lower $f$ values, as well as activity window phase drifts between $f$ bands. The widening of the activity window is primarily due to the increase of $\xi_{f}$ at lower $f$ bands, while the phase drift is primarily due to the higher magnetic polar angle $\delta_{\text {obs }}$ values at lower $f$ bands (see Section 2.1 for discussion). The roughly monochromatic bust rate with spin phase for the slow magnetar model is because the event rate is assumed to be uniform over the NS spin phase: dropping this assumption can lead to activity windows that are not monochromatic. We conclude that the dynamical model of a magnetar with a slow rotation period, as well as a magnetar undergoing free precession, are capable of causing periodic emission consistent with observations of FRB 180916.

The bottom right panel of Figure 5 displays the activity windows for the model describing a magnetar undergoing forced precession. Although these parameters clearly show a widening activity window with a lower $f$ band, the histogram also displays no activity window phase shift with frequency. The lack of activity window phase shift is not unique to these particular model parameters, but is rather a general feature of the forced precession model. Consider a magnetar undergoing forced precession, with LOS direction $\hat{\boldsymbol{n}}=\sin \theta_{n} \hat{\boldsymbol{x}}_{i}+\cos \theta_{n} \hat{\boldsymbol{z}}_{i}$. Because $\hat{\boldsymbol{n}} \cdot \hat{\boldsymbol{m}}_{f}\left(\varphi_{\omega}, \varphi_{n}\right)=\hat{\boldsymbol{n}} \cdot \hat{\boldsymbol{m}}_{f}\left(-\varphi_{\omega},-\varphi_{n}\right)$ (see Equations (14) and (16)), the activity window is always symmetric about $\varphi_{\omega}=$ $\varphi_{n}=0$, irrespective of the emission frequency $f$. Hence, the observed activity window phase drift with $f$ in FRB 180916 disfavors the dynamical model of a magnetar undergoing forced precession.

\section{Proposed Observational Tests}

The previous section shows the dynamical model of a magnetar with a slow rotation period, and a magnetar undergoing free precession, could explain the polarization and frequency-dependent activity window of FRB 180916, while the magnetar undergoing forced precession was disfavored. In this section, we describe how further polarization and activity window measurements can refine constraints on model parameters, and additional observations can favor or rule out the remaining dynamical theories to explain periodic FRBs.

If additional periodic FRB sources are detected, the PA swings and activity windows are expected to change from 

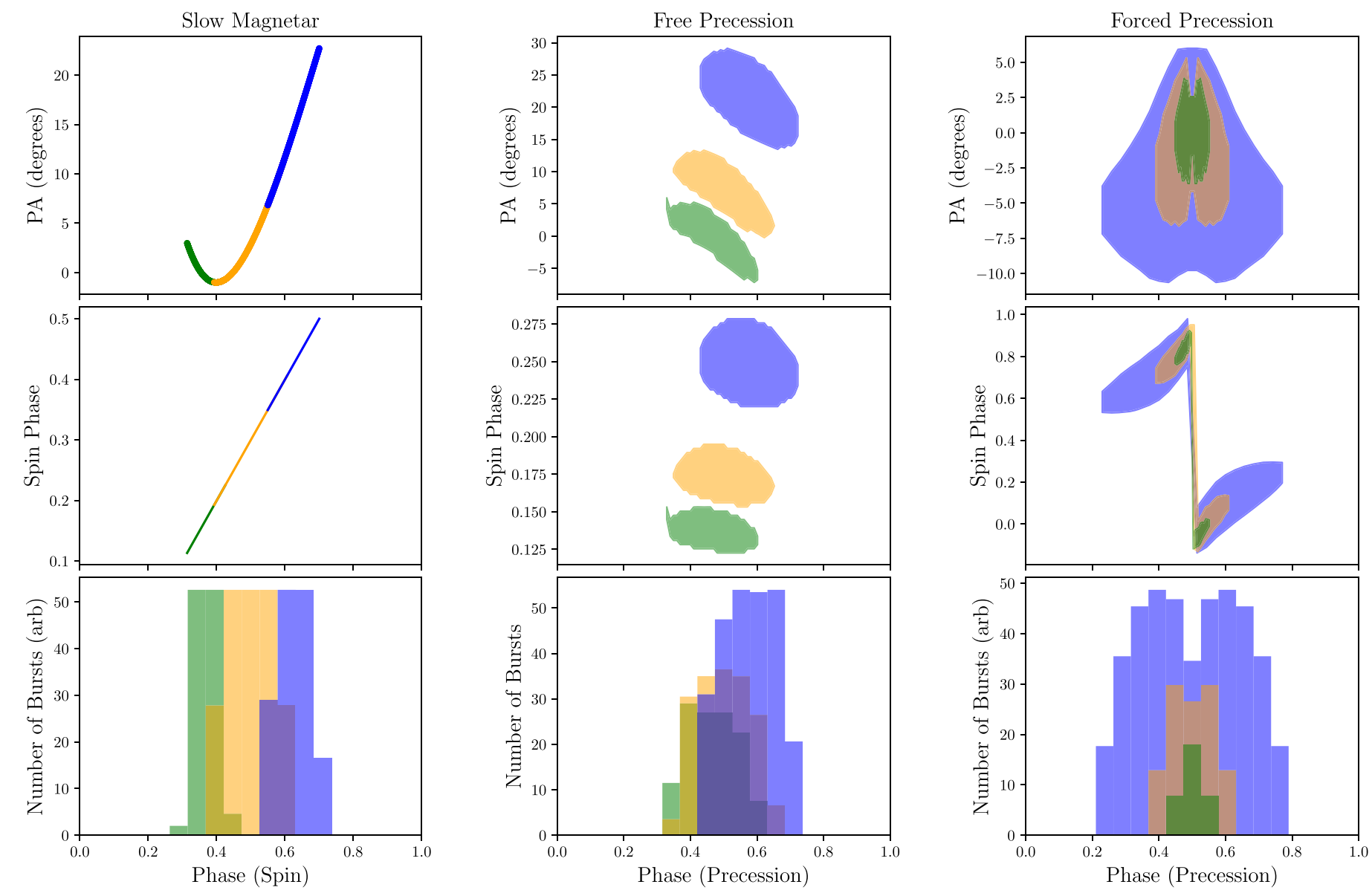

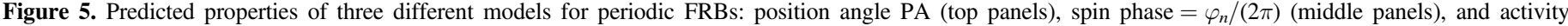

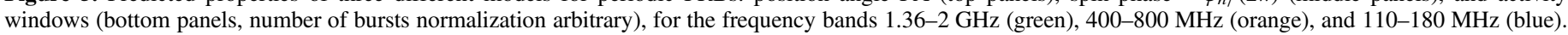

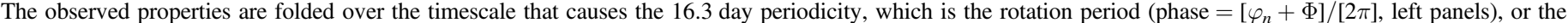

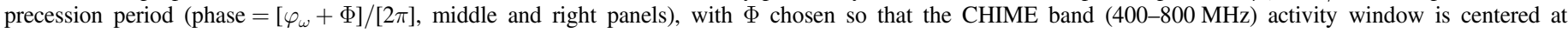

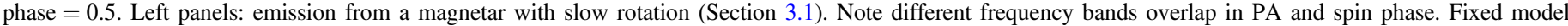

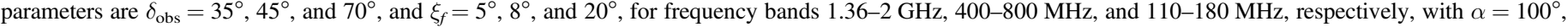

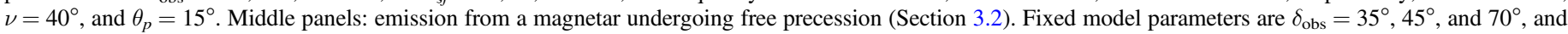

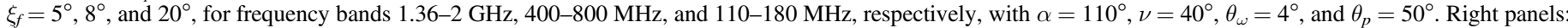

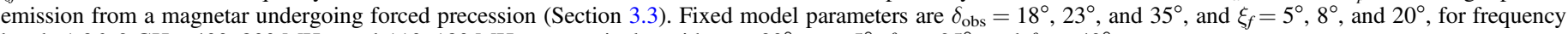
bands $1.36-2 \mathrm{GHz}, 400-800 \mathrm{MHz}$, and 110-180 MHz, respectively, with $\alpha=30^{\circ}, \chi=5^{\circ}, \theta_{\omega}=25^{\circ}$, and $\theta_{n}=40^{\circ}$.

source to source. Within the context of our phenomenological emission model, the phase drift of the FRB activity window with frequency results from a change of emission region. This phase drift can be in either direction, depending on the direction of rotation/precession, as well as the orientation of the emission regions with respect to each other. Event rates of FRBs can also differ significantly across frequency due to different magnetic field geometries. However, both the slowly rotating and freely precessing magnetars require the emission regions to be asymmetric with respect to the NS dipole axis. In contrast, it is difficult for the induced precession model to have an activity window that drifts asymmetrically in phase with frequency.

All dynamical models allow a small PA change with precession or spin phase, while larger PA changes like in the case of FRB 180301 (Luo et al. 2020) are also allowed. The PA variations of both models differ between frequency bands. However, for a slowly rotating magnetar, there is a constant PA angle at each (spin) phase, while for the freely precessing magnetar, a range of PA values are expected at each (precession) phase (Figure 5, upper panels). Moreover, for a fixed precession phase, only certain spin phases will lead to an observed burst (Figure 5, middle panels). More accurate PA measurements from high signal-to-noise ratio bursts would be able to distinguish between these two models (or rule out both).

The precession model also requires a fixed emission region, and a small timescale periodicity due to the rotation period of the magnetar. This short periodicity may be concealed by various timing noises common in young magnetars. However, if a short period is found, the precession model predicts a change of the short periodicity duty cycle with the NS precession phase. For instance, in Figure 5, central middle panel, the spin duty cycle appears smaller at the edge of each active (precession) phase. Moreover, the active window in the spin phase is changing against frequency with this setup of asymmetric emission against magnetic pole.

To get the same activity windows, the free-precession model requires a smaller emission region than the slowly rotating magnetar model. This is because for each spin phase of the slowly rotating magnetar, the LOS points at a specific location on the NS surface, while for each precession phase of the freely precessing magnetar, the LOS rotates around the NS rotation axis. Distinguishing between these two models would constrain the FRB emission region altitude and angular size. 
Recently, Katz (2021) discussed how detecting a change in the FRB period can further differentiate between dynamical theories. We note the freely precessing magnetar model expects a much larger period change than the slowly rotating magnetar model, since the spin-down timescale is much shorter in the former model due to the faster rotation frequency (e.g., Zanazzi \& Lai 2020).

\section{Summary and Conclusions}

In this Letter, we construct a phenomenological model of FRB emission from rotating magnetars to test periodic FRB theories, in light of recent measurements of small polarization PA swings during and between bursts, as well as the narrowing and phase shift of burst activity windows with frequency. Our model assumes bursts are emitted from a region anchored into the magnetar and offset from the dipole axis, with region size and dipole offset angle increasing with a decrease in frequency, due to the altitude dependence of curvature radiation on frequency (Section 2.1). The model PA values are given by the rotating vector model (Section 2.2).

Using this model, we constrain three separate dynamical models that have been invoked to explain the 16.3 day periodicity of FRB 180916: a magnetar with a slow rotation period (Section 3.1), a magnetar undergoing free precession (Section 3.2), and a magnetar undergoing forced precession due to an external body (Section 3.3). We find the slowly rotating and freely precessing magnetar models can produce PA swings and frequencydependent activity windows consistent with recent observations, but the magnetar undergoing forced precession is disfavored as a dynamical model, due to its inability to produce a frequencydependent phase drift of the burst activity window (Section 4). Future observations are necessary to distinguish between the remaining theories, and understand what is causing the periodicity of FRB 180916 (Section 6)

D.Z.L. acknowledges the discussion with Liam Connor and Vikram Ravi. J.Z. was supported by the Natural Sciences and Engineering Research Council of Canada (NSERC), [funding reference \#CITA 490888-16].

\section{ORCID iDs}

Dongzi Li (i) https://orcid.org/0000-0001-7931-0607 J. J. Zanazzi (i) https://orcid.org/0000-0002-9849-5886

\section{References}

Beniamini, P., Wadiasingh, Z., \& Metzger, B. D. 2020, MNRAS, 496, 3390 Beskin, V. S., \& Philippov, A. A. 2012, MNRAS, 425, 814 Bilous, A. V., Watts, A. L., Harding, A. K., et al. 2019, ApJL, 887, L23 Bochenek, C. D., Ravi, V., Belov, K. V., et al. 2020, Natur, 587, 59 Chatterjee, S., Law, C. J., Wharton, R. S., et al. 2017, Natur, 541, 58
CHIME/FRB Collaboration, Amiri, M., Andersen, B. C., et al. 2020a, Natur, 582,351

CHIME/FRB Collaboration, Andersen, B. C., Bandura, K., et al. 2019, ApJL, $885, \mathrm{~L} 24$

CHIME/FRB Collaboration, Andersen, B. C., Bandura, K. M., Bhardwaj, M., et al. 2020b, Natur, 587, 54

Cordes, J. M. 1978, ApJ, 222, 1006

Cordes, J. M., \& Wasserman, I. 2016, MNRAS, 457, 232

Cruces, M., Spitler, L. G., Scholz, P., et al. 2021, MNRAS, 500, 448

Dai, Z. G., Wang, J. S., Wu, X. F., \& Huang, Y. F. 2016, ApJ, 829, 27

Fonseca, E., Andersen, B. C., Bhardwaj, M., et al. 2020, ApJL, 891, L6

Goldreich, P., \& Julian, W. H. 1969, ApJ, 157, 869

Ioka, K., \& Zhang, B. 2020, ApJL, 893, L26

Kalapotharakos, C., Wadiasingh, Z., Harding, A. K., \& Kazanas, D. 2021, ApJ, 907, 63

Kaspi, V. M., \& Beloborodov, A. M. 2017, ARA\&A, 55, 261

Katz, J. I. 2014, PhRvD, 89, 103009

Katz, J. I. 2021, MNRAS, 502, 4664

Kumar, P., Lu, W., \& Bhattacharya, M. 2017, MNRAS, 468, 2726

Levin, Y., Beloborodov, A. M., \& Bransgrove, A. 2020, ApJL, 895, L30

Lorimer, D. R., Bailes, M., McLaughlin, M. A., Narkevic, D. J., \& Crawford, F. 2007, Sci, 318, 777

Lu, W., \& Kumar, P. 2018, MNRAS, 477, 2470

Lu, W., Kumar, P., \& Narayan, R. 2019, MNRAS, 483, 359

Lu, W., Kumar, P., \& Zhang, B. 2020, MNRAS, 498, 1397

Luo, R., Wang, B. J., Men, Y. P., et al. 2020, Natur, 586, 693

Lyutikov, M. 2020, ApJ, 889, 135

Lyutikov, M., Barkov, M. V., \& Giannios, D. 2020, ApJL, 893, L39

Manchester, R. N., \& Taylor, J. H. 1977, Pulsars (San Francisco, CA: W. H. Freeman and Company)

Marcote, B., Nimmo, K., Hessels, J. W. T., et al. 2020, Natur, 577, 190

Michilli, D., Seymour, A., Hessels, J. W. T., et al. 2018, Natur, 553, 182

Nimmo, K., Hessels, J. W. T., Keimpema, A., et al. 2020, arXiv:2010.05800

Pastor-Marazuela, I., Connor, L., van Leeuwen, J., et al. 2020, arXiv:2012. 08348

Philippov, A. A., \& Spitkovsky, A. 2018, ApJ, 855, 94

Phillips, J. A. 1992, ApJ, 385, 282

Pleunis, Z., Michilli, D., Bassa, C. G., et al. 2020, arXiv:2012.08372

Radhakrishnan, V., \& Cooke, D. J. 1969, ApL, 3, 225

Rajwade, K. M., Mickaliger, M. B., Stappers, B. W., et al. 2020, MNRAS, 495, 3551

Rankin, J. M. 1993, ApJ, 405, 285

Riley, T. E., Watts, A. L., Bogdanov, S., et al. 2019, ApJL, 887, L21

Ruderman, M. A., \& Sutherland, P. G. 1975, ApJ, 196, 51

Sob'yanin, D. N. 2020, MNRAS, 497, 1001

Spitler, L. G., Cordes, J. M., Hessels, J. W. T., et al. 2014, ApJ, 790, 101

Spitler, L. G., Scholz, P., Hessels, J. W. T., et al. 2016, Natur, 531, 202

Storch, N. I., Ho, W. C. G., Lai, D., Bogdanov, S., \& Heinke, C. O. 2014, ApJL, 789, L27

Tchekhovskoy, A., Philippov, A., \& Spitkovsky, A. 2016, MNRAS, 457, 3384

Tendulkar, S. P., Gil de Paz, A., Kirichenko, A. Y., et al. 2021, ApJL, 908, L12

Tong, H., Wang, W., \& Wang, H.-G. 2020, RAA, 20, 142

Wadiasingh, Z., \& Timokhin, A. 2019, ApJ, 879, 4

Wang, C., Lai, D., \& Han, J. 2010, MNRAS, 403, 569

Wang, W., Zhang, B., Chen, X., \& Xu, R. 2019, ApJL, 876, L15

Weisberg, J. M., Everett, J. E., Cordes, J. M., Morgan, J. J., \& Brisbin, D. G. 2010, ApJ, 721, 1044

Yang, H., \& Zou, Y.-C. 2020, ApJL, 893, L31

Yang, Y.-P., \& Zhang, B. 2018, ApJ, 868, 31

Yang, Y.-P., Zhu, J.-P., Zhang, B., \& Wu, X.-F. 2020, ApJL, 901, L13

Zanazzi, J. J., \& Lai, D. 2015, MNRAS, 451, 695

Zanazzi, J. J., \& Lai, D. 2020, ApJL, 892, L15 